In press, 22 November 2018, Biological Conservation

\title{
Is habitat fragmentation bad for biodiversity?
}

Lenore Fahrig, Víctor Arroyo-Rodríguez, Joseph R. Bennett, Véronique Boucher-Lalonde, Eliana Cazetta, David J. Currie, Felix Eigenbrod, Adam T. Ford, Susan P. Harrison, Jochen A.G. Jaeger, Nicola Koper, Amanda E. Martin, Jean-Louis Martin, Jean Paul Metzger, Peter Morrison, Jonathan R. Rhodes, Denis A. Saunders, Dan Simberloff, Adam C. Smith, Lutz Tischendorf, Mark Vellend, James I. Watling

\begin{abstract}
In a review of landscape-scale empirical studies, Fahrig (2017a) found that ecological responses to habitat fragmentation per se (fragmentation independent of habitat amount) were usually non-significant (> 70\% of responses) and that $76 \%$ of significant relationships were positive, with species abundance, occurrence, richness, and other response variables increasing with habitat fragmentation per se. Fahrig concluded that to date there is no empirical evidence supporting the widespread assumption that a group of small habitat patches generally has lower ecological value than large patches of the same total area. Fletcher et al. (2018) dispute this conclusion, arguing that the literature to date indicates generally negative ecological effects of habitat fragmentation per se. They base their argument largely on extrapolation from patchscale patterns and mechanisms (effects of patch size and isolation, and edge effects) to landscape-scale effects of habitat fragmentation. We argue that such extrapolation is unreliable because: (1) it ignores other mechanisms, especially those acting at landscape scales (e.g., increased habitat diversity, spreading of risk, landscape complementation) that can counteract effects of the documented patch-scale mechanisms; and (2) extrapolation of a small-scale mechanism to a large-scale pattern is not evidence of that pattern but, rather a prediction that must be tested at the larger scale. Such tests were the subject of Fahrig's review. We find no support for Fletcher et al.'s claim that biases in Fahrig's review would alter its conclusions. We encourage further landscape-scale empirical studies of effects of habitat fragmentation per se, and research aimed at uncovering the mechanisms that underlie positive fragmentation effects.
\end{abstract}

Key words: corridors; cross-scale extrapolation; edge effect; habitat diversity; habitat fragmentation per se; habitat loss; landscape configuration; landscape heterogeneity; patch isolation; patch size; SLOSS; spatial scale

\section{Introduction}

In their critique, "Is habitat fragmentation good for biodiversity?", Fletcher et al. (2018) object to the methods, results, and conclusions presented in a review by Fahrig (2017a). Fahrig (2017a) reviewed the empirical evidence for ecological responses to habitat fragmentation per se, i.e. habitat fragmentation independent of habitat amount. She found that responses were usually non-significant (> $70 \%$ of responses), and of the significant responses, 76\% increased with habitat fragmentation per se. Response variables included, in decreasing order of prevalence, the occurrence/abundance of individual species, the richness/abundance of species groups, the movement/demographic success of individual species, and water quality measures. Fahrig (2017a) also categorized the responses by species conservation status, taxonomic group, biome, and various study attributes. In all cases there were more significant positive responses to habitat fragmentation per se than negative responses. 
Fletcher et al. (2018) argue that Fahrig's (2017a) results are biased, and that the conclusions drawn from the results are dangerous for conservation. We discuss these claims in separate sections below. But first we describe a fundamental problem in the reasoning of Fletcher et al. (2018). Fletcher et al. (2018) take the occurrence of particular patterns and mechanisms observed at the patch scale (effects of habitat patch size and isolation, and edge effects) as evidence for negative fragmentation effects at landscape scales. This reasoning is flawed because: (1) it ignores other mechanisms that can cause positive responses to fragmentation per se (e.g., reduced competition, increased habitat diversity, higher between-patch movement success), which may counteract effects of the documented mechanisms; and (2) relationships between mechanisms and patterns at one scale cannot be simply extrapolated to other scales. Such extrapolations are predictions that must be tested. To avoid inaccurate predictions of pattern from mechanism, scientists proceed either by: (1) first documenting a pattern and then conducting further study to elucidate the mechanism(s) responsible for that pattern; or (2) establishing potential mechanisms, building a model to predict a pattern from these mechanisms, and then testing this prediction. Such tests are critical because even highly intuitive mechanisms may not produce the predicted pattern in nature, especially when the prediction involves cross-scale inference. We elaborate on this important point in the next section.

\section{Extrapolation constitutes prediction, not evidence}

Fahrig (2017a) reviewed the empirical evidence for effects of habitat fragmentation per se on ecological response variables. As explained by Fahrig (2003, 2017a), habitat fragmentation per se is a concept that applies specifically at landscape scales, and not to individual patches. Although Fletcher et al. (2018) do not challenge this definition, many of their comments suggest a mixing of patch and landscape scales in their conceptualization of fragmentation per se. For example, while they refer to "fragmented landscapes," they also state that, "most species now live in fragmented patches." As the word "fragmented" means "broken apart," by definition a single patch cannot be described as fragmented.

To avoid possible confusion, here we reiterate the definition of habitat fragmentation per se and explain why this concept applies at landscape scales and not at a patch scale (see also Fahrig, 2003; 2017a). When natural land cover is converted to anthropogenic land cover ("habitat loss"), the remaining natural land cover is often fragmented into a greater number of smaller patches. "To fragment" literally means "to break apart," so fragmentation refers specifically to an increase in the number of patches, which often accompanies habitat loss. Although habitat removal often causes habitat fragmentation, habitat removal does not necessarily entail habitat fragmentation. For example, if a single large area of habitat is diminished, this is not habitat fragmentation because the number of patches has not increased; the habitat has not been broken apart. Similarly, habitat fragmentation does not occur when a whole habitat patch is removed from a landscape, because the number of patches has not increased, but rather decreased.

For a given loss of habitat, the extent of fragmentation can vary greatly among landscapes of a given size (e.g., De Camargo et al., 2018), resulting in a few large patches in some landscapes and many small patches in others (Fig. 1: A vs. B, or C vs. D). Does it generally matter to species occurrence, abundance, or richness whether habitat is fragmented into many small or few large patches? In other words, do large patches generally have a higher ecological value than groups of small patches totalling the same area? 


\section{[Figure 1]}

Note that these questions cannot be addressed by asking, for example, whether a single small patch has lower species richness than an equal-area portion of a large patch, a question asked in some studies that Fletcher et al. (2018) cite. If, for example, we find that a single small patch contains fewer species than an equal-area portion of a large patch, it can still be the case - and indeed often is the case (Fahrig, 2017a) that many small patches collectively harbour more species than a few large patches of the same total area. This results from high beta diversity across the small patches (Tscharntke et al., 2012). In this situation, removing a small patch would on average have a larger impact on total species richness than removing an equal-sized area from a large patch, even though the species richness of a small patch may be lower than the species richness in an equal sized area in a large patch. In other words, the patch-scale result need not scale up to a landscape.

The question, "Do large patches generally have a higher ecological value than groups of small patches totalling the same area?" is important for conservation. If fragmentation, independent of habitat amount (fragmentation per se), has widespread negative effects on species abundance, occurrence, and richness, then conservation should focus on preserving large, contiguous habitat areas. If fragmentation per se has negligible effects on species then conservation efforts should focus on all habitat, regardless of the sizes of the patches in which it is distributed. If fragmentation per se has widespread positive effects on species then conservation efforts should focus on preserving a large number of small patches. Note that in all cases the primary goal is to conserve habitat and the species and ecological processes housed therein. To be as clear as possible, we emphasize that in no sense are we arguing that converting a landscape with continuous habitat cover into a landscape of small, isolated habitat patches would be good for the species that rely on that habitat, because this would involve a reduction of the total amount of habitat. But understanding the potential role of fragmentation per se is important to conservation, as has been recognized for decades (e.g., Simberloff and Abele, 1982).

It is quite straightforward in principle to answer the question, "Does it matter to a species or a group of species whether their habitat is fragmented into many small or few large patches?" This requires measuring the ecological response variable, e.g., species occurrence, abundance, or richness, in different landscapes with different levels of habitat fragmentation (Fig. 2D). To measure the level of fragmentation of a landscape we measure the number or density of habitat patches, or highly related metrics (e.g., Jaeger, 2000; see list of search terms in Fahrig, 2017a and below), where habitat is defined according to the requirements of the particular species or species group. As noted by Fletcher et al. (2018), fragmentation is often inversely correlated with habitat amount across randomly selected landscapes. If this correlation is high, then landscapes should be selected to minimize that correlation across the set of sample landscapes (e.g., Ethier and Fahrig, 2011; Trzcinski et al., 1999). Habitat amount can then be included in models along with other covariates such as geographic position, time since habitat removal, or landscape matrix attributes such as road density, agricultural intensity, or matrix heterogeneity, to control for these effects in estimating the effects of habitat fragmentation per se. Although Fletcher et al. (2018) correctly point out that some statistical techniques (e.g., residual regression) are limited in their ability to discriminate habitat fragmentation from other effects, other techniques such as standardized partial regression coefficients (Smith et al., 2009) or structural equation models (Grace 2006) yield unbiased 
estimates of the effects of habitat fragmentation per se, even when it is moderately correlated with habitat amount.

[Figure 2]

A great deal of research has been conducted on species-habitat relationships by comparing across multiple patches rather than multiple landscapes. These patch-scale studies measure ecological responses to patch size, isolation, and distance to edge, providing an enormous amount of information about patch-scale effects on a wide variety of organisms. If a conservation effort were concerned with an individual patch, the results of these studies could help guide decisions. However, they cannot guide decisions about the conservation value of sets of large patches vs. small patches totalling the same area. Thus, our key concern here is that patch-scale results have frequently been used to make inferences about the ecological effects of habitat fragmentation per se (e.g., third column of Table 1 in Fletcher et al., 2018), which is a landscape-scale phenomenon. While patterns at landscape scales might be determined mainly by patchscale processes, there is no logical reason to infer that they must be. That is a hypothesis that must be tested.

Pitfalls in extrapolation across scales are well documented in ecology. For example, biodiversity responses to a variety of natural and anthropogenic drivers vary across spatial scales, and sometimes switch directions from one spatial scale to another (Chase et al. 2018). As first pointed out by Levine and D'Antonio (1999), diversities of native and exotic plants are often negatively correlated at a small scale, but they are usually positively correlated at larger scales. Similarly, human presence often correlates negatively with species richness at small spatial scales, but positively at larger scales (Pautasso, 2007). The same is true for temporal biodiversity change: trends at small scales cannot necessarily be extrapolated to larger scales (Jarzyna and Jetz, 2018). In short, we should not be surprised to find that results at the patch scale do not permit reliable predictions at landscape scales.

In the context of habitat fragmentation effects, predictions from patch-scale studies to landscape-scale fragmentation effects may be wrong for two reasons. First, patch-scale studies confound habitat fragmentation with habitat amount: a larger patch has more habitat, and a more isolated patch is more isolated exactly because there is less habitat nearby. It is therefore not logically possible to extrapolate from effects of patch size and isolation to landscape-scale effects of habitat fragmentation controlling for habitat amount (fragmentation per se). Confounding of habitat fragmentation with habitat amount may partially explain why Fletcher et al. (2018) argue that the effects of habitat fragmentation per se cannot be estimated using standard statistical approaches. They argue that habitat fragmentation effects cannot be separated from habitat amount effects owing to the inter-linked nature of the mechanisms that underlie them both. This argument makes sense at the patch scale: if studies were conducted only at the patch scale, then it would indeed be impossible to partition variance between habitat fragmentation and habitat amount. It is precisely for this reason that efforts to quantify effects of habitat fragmentation per se - the focus of Fahrig (2017a) - study replicate landscapes, rather than replicate patches. When observations are replicated across landscapes, it is fairly straightforward to estimate empirically the effects of fragmentation per se using standard statistical methods, so long as replicate landscapes can be selected such that the correlation between habitat amount and habitat fragmentation is not too high (e.g., Trzcinski et al., 1999; Ethier and Fahrig 2011; De Camargo et al., 2018). 
The second reason that patch-scale studies do not provide evidence for effects of fragmentation per se is that mechanisms shown to influence patch-scale species occurrence, abundance, or richness (e.g, distance to edge) may be counteracted by other mechanisms influencing these ecological responses at landscape scales (also discussed by Arroyo-Rodriguez et al., 2017). For example, if we show that a particular species avoids habitat edges, then we might predict that the species will have lower occurrence or abundance in a landscape containing more edge (i.e., a more fragmented landscape, as edge density and number of patches are usually correlated) than in a landscape containing less edge. The argument is that negative edge effects mean negative fragmentation effects. This extrapolation across scales is repeatedly argued by Fletcher et al. (2018), and is one of the key reasons that they cast doubt on the results of Fahrig (2017a). However, this argument is a hypothesis about landscape-scale patterns; it is not evidence. A landscape-scale test of this hypothesis, comparing species occurrence or abundance across different landscapes with different levels of fragmentation, might show no effect of fragmentation, or even a positive effect because of other mechanisms (e.g., increased movement success, decreased inter-species competition, increased landscape complementation) that counteract the edge effect (Fig. 3). As a case in point, based on documented negative edge effects on a variety of taxa in the tropics (Laurance et al., 2002), Fahrig (2003) predicted that ecological effects of fragmentation per se would be generally negative in the tropics. But tests of this prediction did not support it: Fahrig (2017a) found that 92 of 126 (73\%) significant responses to habitat fragmentation per se in the tropics and subtropics were positive (Fig. 11a in Fahrig, 2017a). Thus, the patch-scale mechanism (negative edge effects) did not translate to the predicted landscape-scale pattern. So, even if negative edge effects are as widespread as suggested by Fletcher et al. (2018), this does not necessarily lead to widespread negative effects of habitat fragmentation per se. Habitat fragmentation research should thus move from patch-scale to landscapescale investigation of mechanisms, to understand the landscape-scale patterns better. These patterns may result from a complex interaction among multiple mechanisms, or they may result from rather simple processes (Prevedello et al., 2016). The answer awaits further study.

\section{[Figure 3]}

To summarize this point: to determine whether a widespread small-scale mechanism creates a particular widespread large-scale pattern we need to first demonstrate that the large-scale pattern actually exists, and then to conduct studies aimed at identifying the mechanisms underlying this pattern. In contrast to this approach, the empirical literature described by Fletcher et al. (2018) as addressing "habitat fragmentation" over the past 30 years consists largely of patch-scale studies that demonstrate the occurrence of particular patch-scale patterns and mechanisms, and then extrapolate these to an as-yet undocumented landscapescale pattern of negative fragmentation effects (Fig. 2).

Fahrig (2017a) found that responses to fragmentation per se are usually non-significant, and when they are significant, positive responses are three times as common as negative responses. This suggests that other mechanisms, leading to positive fragmentation effects, counteract and can even outweigh the mechanisms leading to negative effects. Many mechanisms might in fact lead to positive effects of habitat fragmentation per se at landscape scales (Fig. 3). In her review, Fahrig (2017a) generated a preliminary list of such mechanisms by documenting the explanations suggested by authors who actually found positive fragmentation effects. Several of these mechanisms have been known in the ecological literature 
for many decades, e.g., spreading of risk, reduced competition, and stabilization of predator-prey interactions.

Note that we do agree with Fletcher et al. (2018) that elucidation of mechanisms is important and that more research is needed to connect patterns and mechanisms at multiple scales (see also Soranno et al., 2014). That said, we suggest that it is not helpful to document a mechanism, and then insist that the patterns predicted from that mechanism must occur, irrespective of the empirical evidence. The empirical evidence summarized in Fahrig (2017a) does not support the prediction of generally strong, negative fragmentation effects at landscape scales. Rather, the pattern indicates that relationships are usually nonsignificant, and when they are significant they are more often positive than negative. Research efforts to elucidate mechanisms should focus on those that explain the patterns that are actually observed. Thus, evidence that positive effects of fragmentation are more common than negative effects (Fahrig, 2017a) should motivate us to focus more attention on the potential underlying mechanisms of these positive effects.

In contrast to this approach, Fletcher et al. (2018) attempt to infer effects of fragmentation per se at landscape scales from particular patch scale patterns and mechanisms. As these predictions are not supported by the studies reviewed by Fahrig (2017a), Fletcher et al. (2018) conclude that the data in Fahrig (2017a) must be biased, i.e., that Fahrig (2017a) excluded studies showing the opposite. However, while Fletcher et al. (2018) suggest potential sources of bias, they do not show that addressing these would actually change the results of Fahrig (2017a), as explained in the next section.

\section{$\underline{\text { Bias }}$}

The main "bias" identified by Fletcher et al. (2018) is that Fahrig's (2017a) review does not include patchscale studies "that are highly relevant to the questions addressed." These include studies on the effects of patch size, patch isolation, corridors, or edges. Fletcher et al. (2018) argue that these studies constitute the bulk of the "fragmentation" literature. We agree that these studies make significant contributions to understanding patch-scale phenomena. However, as explained by Fahrig (2017a) and above, these patchscale studies do not actually test for effects of habitat fragmentation per se. The results from these studies have been extrapolated to make predictions about fragmentation per se, but they do not provide evidence for those predictions. Therefore they were excluded from Fahrig (2017a), which was focussed exclusively on evidence at landscape scales.

Fletcher et al. (2018) also argue that by omitting theoretical studies, Fahrig (2017a) excluded important evidence about the effects of habitat fragmentation per se on ecological responses. However, mechanistic modelling studies make predictions of what we would see in nature if their assumptions hold, and if the factors that are excluded from the model do not influence nature. Models do not provide evidence of what actually does happen in nature, except insofar as they successfully predict patterns observed in nature. Just as with patch-scale studies, the predictions from modeling studies must be tested by studying ecological responses across actual landscapes varying in their level of habitat fragmentation. The objective of Fahrig (2017a) was to review such empirical landscape-scale tests of the ecological effects of habitat fragmentation per se. 
Fletcher et al. (2018) also state that the search terms used by Fahrig (2017a) led to a biased review. As mentioned by Fletcher et al. (2018), a vast and growing number of studies use the term "fragmentation." They also correctly point out that very few of the studies that estimate the effect of habitat fragmentation independent of habitat amount actually use the term "fragmentation per se." However, they incorrectly state that "habitat fragmentation per se" was the only search term used by Fahrig (2017a). In fact, the approach in Fahrig (2017a) was to identify an extensive set of search terms related to the methods that researchers use to estimate fragmentation effects independent of habitat amount. The search terms were: "fragmentation per se" OR "SLOSS” OR [(“edge density" OR "edge length" OR "number of patches" OR "mean patch size" OR "boundary length" OR "patch density" OR "median patch size” OR "clumping index" OR "splitting index" OR "aggregation index" OR "like adjacencies" OR "fractal dimension" OR "IJI" OR "mean circumscribing circle" OR "largest patch index" OR "shape index" OR "mean core area" OR "proportion core area" OR "mean nearest-neighbor" OR "mean perimeter to area" OR "mean edge to area") AND ("habitat" OR "forest" OR "grassland" OR "wetland” OR "coral” OR "landscapes" OR "watersheds" OR "catchments")] (Fahrig, 2017a).

Fletcher et al. (2018) are particularly concerned that Fahrig's (2017a) review did not include the seven "fragmentation" experiments conducted by several of the authors of Fletcher et al. (2018). These impressive long-term experiments have produced a wide range of findings on effects of habitat loss and a variety of patch-scale patterns and processes. However, Fahrig (2017a) omitted them because, to date, the analyses that have been conducted using the data from these experiments do not actually test for effects of fragmentation independent of habitat amount by comparing responses across landscapes. Study designs that control for habitat amount meet at least one of three conditions: (1) replicate landscapes in the study have the same amount of habitat, but varying amounts of fragmentation; or (2) there is little correlation between habitat fragmentation and habitat amount across landscapes; or (3) effects of landscape-scale habitat amount are included in statistical analyses, to isolate the effects of fragmentation (e.g., Trzcinski et al., 1999; Ethier and Fahrig 2011; De Camargo et al., 2018). Fletcher et al. (2018) specifically mention the omission of the results of Gonzalez et al. (1998) by Fahrig (2017a). In addition to comparing a continuous landscape to a fragmented landscape containing four small patches and much less total habitat (i.e., not fragmentation per se), Gonzalez et al. (1998) also compared species abundances in patches within a corridor treatment to those in patches within a pseudo-corridor treatment with breaks in the corridors. Here, each treatment contained four patches and approximately the same total habitat area. This comparison could indeed be used to evaluate effects of fragmentation per se if the data were analyzed at the landscape scale. Given that one can never be certain of having comprehensively found all relevant studies, no doubt there are additional studies that could be added to the database in Fahrig (2017a). Importantly, however, Fletcher et al. (2018) provide no evidence at all that the studies in Fahrig (2017a) were biased systematically with respect to the direction or magnitude of the effect of fragmentation per se, as we describe in subsequent paragraphs.

Fletcher et al. (2018) identify various other shortcomings of the methods in Fahrig (2017a), which they say could "bias" the results. However, they do not explain how these shortcomings would lead to a bias specifically in favour of positive significant fragmentation effects. For example, they point out that Fahrig (2017a) used a simple vote-counting approach rather than a full meta-analytic approach. This is a fair criticism, and one that Fahrig (2017a) discussed. However, Fletcher et al. (2018) provide no evidence or 
argument for why vote-counting would specifically favour positive significant responses to fragmentation per se. Such a bias seems highly unlikely.

Fletcher et al. (2018) also argue that Fahrig's (2017a) results are biased because she did not consider time since fragmentation. However, Fletcher et al. do not state the process by which they expect time since fragmentation to produce a bias in favour of positive effects of fragmentation per se. Perhaps they assume that positive fragmentation effects occur immediately following habitat removal, and that these effects then become negative over time. For this to create a bias in favour of positive fragmentation effects in Fahrig's (2017a) review, the habitat removal that created the fragmentation gradients in the studies finding negative fragmentation effects would need to have occurred longer ago than the habitat removal that created the fragmentation gradients in the studies that found positive effects. As most of the studies in Fahrig (2017a) do not contain information on the time since habitat removal, this assumption cannot be directly evaluated. Here we also note that Fletcher et al.'s comment conflates the time since fragmentation with the time over which a system is studied, in that they criticize the multi-landscape observational studies in Fahrig (2017a) as representing only "snapshots" in time. This criticism ignores the fact that most of these snapshots were taken many decades after habitat removal, so short-term effects have likely dissipated in many of the study systems Fahrig (2017a) reviewed. Nevertheless, we do agree that the potential role of time since fragmentation requires further investigation.

Fletcher et al. (2018) also object to the inclusion of SLOSS-type studies, citing critiques by Ramsey (1989) and Mac Nally and Lake (1999) of Quinn and Harrison (1988). However, these papers specifically criticize the saturation index, not the SLOSS accumulation curve introduced by Quinn and Harrison (1988). Fahrig (2017a) used the curves, not the saturation index, to estimate the directions of the SLOSS study results. Fletcher et al. (2018) note that there is no test for statistical significance of SLOSS accumulation curves. This is true, and for this reason, Fahrig (2017a) included only completely unambiguous SLOSS results, i.e., those in which either the single-large curve was entirely above the several-small curve or the several-small curve was entirely above the single-large curve (see Fig. 7 in Fahrig, 2017a). Fahrig found 60 such unambiguous cases; in all 60 cases the several small curve was above the single large curve, indicating positive fragmentation effects on species richness. Fletcher et al. (2018) do correctly point out that two curves are missing from Fahrig (2017a), contained in Blake and Karr (1984). From that study, Fahrig (2017a) included only the curve for all bird species combined. Blake and Karr (1984) also have curves for two subgroups, forest interior specialists, which showed a negative effect of fragmentation per se, and long-distance migrants, which showed a positive effect of fragmentation per se. Therefore, the correct count for the SLOSS studies in Fahrig (2017a) is that 61 of 62 significant SLOSS-based fragmentation effects (98\%) are positive. This result does not shift the weight of evidence, and therefore does not influence the conclusions of Fahrig (2017a). We also note that omitting all of the SLOSS studies from Fahrig (2017a) would not affect the conclusions, as 72\% (230 of 321) of the significant fragmentation per se effects from the non-SLOSS studies were positive (Fig. 9a in Fahrig 2017a). Similarly, Fletcher et al.'s argument that inclusion of studies using species richness as a response variable biased the results is not supported. The majority of significant responses were for single-species response variables (mostly species occurrence or abundance) and of these responses, 68\% (158 of 232) were positive (Fig. 9d in Fahrig 2017a). 
Fletcher et al. (2018) also suggest that including some undesired species such as exotics biased the results of Fahrig (2017a). It is true that Fahrig (2017a) did not pre-screen response variables for their conservation value. She also did not reverse the sign of fragmentation responses for undesired species. However, Fahrig's (2017) conclusions regarding conservation are not affected by these decisions. This is because the majority of significant relationships with habitat fragmentation per se were positive for threatened and declining species and for habitat specialist species groups. In fact, a slightly higher proportion of responses were positive for declining species than for increasing species (Fig. 9e in Fahrig, 2017a). In other words, the majority of significant responses indicated increasing habitat conservation value with increasing habitat fragmentation per se. Contrary to the arguments of Fletcher et al. (2018), these results do not contradict patch-scale studies showing, for example, that threatened species are disproportionately edge-sensitive (Pfeiffer et al., 2017), for the reason already described: there is no a priori reason to expect patch-scale mechanisms to scale up to landscapes.

Thus, the claim that the conclusions of Fahrig (2017a) derive from biases in Fahrig's methods is not supported. In fact, Fahrig (2017b) demonstrated that publication bias and confirmation bias likely favour negative effects of fragmentation per se across the studies in Fahrig (2017a). Even with this bias in favour of negative fragmentation effects, the significant effects of fragmentation per se are more often positive than negative.

\section{Conservation}

Fletcher et al. (2018) argue that understanding the influence of habitat fragmentation per se on biodiversity is critically important for conservation. In particular, they state, "it is alarming that Fahrig (2017a) concludes that, 'there is no justification for assigning lower conservation value to small patches than to an equivalent area within a large patch' " (Fletcher et al., 2018). We agree that this debate is important for conservation. But, given the results of Fahrig (2017a), what we find "alarming" for conservation is the nearly complete lack of protection for habitat that is divided into small patches. Around the world, habitat protection almost always emphasizes large, "intact" habitats while ignoring small patches of habitat, even where these may sum to a large total area. For example, most small wetlands have little or no protection (reviewed by Hill et al., 2018). The same is true for small forest patches: forestry policy in Ontario, Canada, recommends cutting patterns that "defragment" the remaining forest by removing small patches (OMNR, 2002); and in Mexico and Costa Rica, landowners and communities can be paid to preserve forest, but only patches larger than 25 ha in Mexico or 2 ha in Costa Rica (Hernández-Ruedas et al., 2014; Oficina Nacional Forestal, 2018). These minima are larger than the majority of remaining forest patches in the tropics (Taubert et al., 2018).

The widespread assumption that fragmentation effects are large and negative has clearly contributed to this lack of concern for conservation of small patches. It has led to the cumulative erosion of natural habitats, one small patch at a time, as the loss of these patches goes unnoticed even in highly degraded landscapes (Arroyo-Rodríguez et al., 2009; Bennett et al., 2013; Tulloch et al., 2016). A first step in halting habitat loss and biodiversity decline is the general recognition that there is no empirical biological justification for a general rule that automatically assigns lower conservation value to small patches than to an equivalent area within large patches. All habitat loss has ecological consequences. 
Of course there may be particular situations where it makes sense to focus conservation efforts on large patches, such as when the target species or species group responds negatively to fragmentation per se. Indeed, Fahrig (2017a) found $24 \%$ of significant responses to fragmentation per se were negative. In addition, in some situations there may be practical reasons to favour large patches, related to other land management objectives, the availability of habitat for protection, and the logistics of protecting a network of small patches. However, none of these qualifications affects the need for evidence-based landscape management decisions.

Thus, the answers to the reciprocal questions posed in the titles of Fletcher et al. (2018) ("Is habitat fragmentation good for biodiversity?") and this response ("Is habitat fragmentation bad for biodiversity?") are both "no" for fragmentation per se, the subject of the review in Fahrig (2017a). Habitat loss, and habitat fragmentation measures that are confounded with habitat loss, generally show negative relationships to biodiversity. However, habitat fragmentation per se, i.e., fragmentation controlling for habitat amount, is neither generally good nor generally bad for biodiversity or other ecological response variables. Overall its effects are usually non-significant (>70\% of responses; Fahrig 2017a), and when they are significant, the relationships are about three times as likely to be positive as negative. In addition, the majority of significant responses are positive for threatened and declining species and for specialist groups of species. Of course this conclusion may change with the accumulation of more empirical studies of habitat fragmentation per se, and so we acknowledge that the characterization of "fragmentation is bad" as a zombie idea (Fahrig, 2017a; b) may be premature. We understand that some readers of Fahrig (2017a; b) may object to the use of this term. We do not see it as an invective, but rather as a challenge, in the context of scientific debate and reflection, to a long-lived perspective that apparently does not hold up under the weight of evidence (Fahrig, 2017a). The data to date suggest that conservation planners should focus on the conservation and restoration of habitat, and on the avoidance and mitigation of other important impacts on species such as high-intensity agriculture and road traffic. As a general rule, approaches that maximize the total amount of habitat conserved, irrespective of its level of fragmentation, will result in the largest ecological benefit.

Finally, we emphasize that we agree with Fletcher et al. (2018) that this debate is important for conservation. We also agree that research is needed to explain "why and when these habitat fragmentation effects occur, how they interact with other human-induced changes, and under what situations fragmentation effects will be positive or negative" (Fletcher et al., 2018). Multiple mechanisms may result in positive or negative landscape-scale responses to habitat fragmentation (Fig. 3), and these may vary with context. Therefore, understanding the mechanisms for a particular effect of fragmentation per se is not a trivial undertaking. Thus, we encourage future work directed at disentangling the effects of those mechanisms on the observed landscape-scale responses to habitat fragmentation.

In closing, we reiterate that documenting a mechanism is not equivalent to demonstrating a pattern predicted from that mechanism, especially when the mechanism and pattern are evaluated at different spatial scales. The goal of Fahrig's (2017a) review was to determine the degree to which a general pattern of negative or positive effects of habitat fragmentation is manifested across existing landscape-scale studies. More work is needed to understand why the significant effects of fragmentation per se are more often positive than negative. At the same time, it is important that conservation practitioners recognize that automatically assigning low conservation value to small habitat patches of equivalent total area to 
In press, 22 November 2018, Biological Conservation

fewer large patches is not justified based on the biological evidence to date, and that in many cases the opposite may be warranted.

\section{Acknowledgements}

LF was funded by the Natural Sciences and Research Council of Canada (NSERC). VAR thanks DGAPA-UNAM for funding his sabbatical stay at the Geomatics and Landscape Ecology Laboratory, Carleton University. EC thanks the Brazilian Coordination for the Improvement of Higher Education Personnel (CAPES grant number 88881.118929/2016-01). ATF was supported by the Canada Research Chairs program. NK was funded by NSERC. The Brazilian National Council for Scientific and Technological Development funded JPM. We are grateful to three anonymous reviewers for helpful comments on the manuscript.

\section{References}

Arroyo-Rodríguez, V., Pineda E., Escobar F., Benítez-Malvido J., 2009. Value of small patches in the conservation of plant-species diversity in highly fragmented rainforest. Conservation Biology 23, 729-739.

Arroyo-Rodríguez, V., Saldaña-Vázquez, R.A., Fahrig, L., Santos, B.A., 2017. Does forest fragmentation cause an increase in forest temperature? Ecological Research 32, 81-88.

Bennett, J.R., Arcese, P., 2013. Human influence and classical biogeographic predictors of rare species occurrence. Conservation Biology 27, 417-421.

Blake, J.G., Karr, J.R., 1984. Species composition of bird communities and the conservation benefit of large versus small forests. Biological Conservation 30, 173-187.

Chase, J.M., McGill, B.J., McGlinn, D.J., May, F., Blowes, S.A., Xiao, X., Knight, T.M., Purschke, O., Gotelli, N.J., 2018. Embracing scale-dependence to achieve a deeper understanding of biodiversity and its change across communities. Ecology Letters 21, 1737-1751.

De Camargo, R.X., Boucher-Lalonde, V., Currie, D.J., 2018. At the landscape level, birds respond strongly to habitat amount but weakly to fragmentation. Diversity and Distributions 5, 629-639.

Ethier, K., Fahrig, L., 2011. Positive effects of forest fragmentation, independent of forest amount, on bat abundance in eastern Ontario, Canada. Landscape Ecology 26, 865-876.

Fahrig, L., 2003. Effects of habitat fragmentation on biodiversity. Annual Reviews of Ecology, Evolution and Systematics 34, 487-515.

Fahrig, L., 2017a. Ecological responses to habitat fragmentation per se. Annual Reviews of Ecology, Evolution and Systematics 48,1-23.

Fahrig L., 2017b. Forty years of bias in habitat fragmentation research. Pp. 32-38 in: Kareiva P., Silliman B., Marvier M. (eds) Effective conservation science: Data not dogma. Oxford University Press, Oxford, UK.

Fletcher, R.J., Didham, R.K., Banks-Leite, C., Barlow, J., Ewers, R.M., Rosindell, J., Holt, R.D., Gonzalez, A., Pardini, R., Damschen, E.I., Melo, F.P.L., Ries, L., Prevedello, J.A., Tscharntke, T., Laurance, W.F., Lovejoy, T., Haddad, N.M., 2018. Is habitat fragmentation good for biodiversity? Biological Conservation 226, 9-15.

Gonzalez, A., Lawton, J.H., Gilbert, F.S., Blackburn, T.M., Evans-Freke, I., 1998. Metapopulation dynamics, abundance, and distribution in a microecosystem. Science 281, 2045-2047. 
Grace, J.B., 2006. Structural equation modeling and natural systems. Cambridge University Press, New York.

Hernández-Ruedas, M.A., Arroyo-Rodríguez, V., Meave, J.A., Martínez-Ramos, M., Ibarra-Manríquez, G., Martínez, E., Jamangapé, G., Melo, F.P.L., Santos, B.A., 2014. Conserving tropical tree diversity and forest structure: the value of small rainforest patches in moderately-managed landscapes. PLoS ONE 9, e98931.

Hill, M., Hassall, C., Oertli, B., Fahrig, L., Robson, B., Biggs, J., Samways, M., Usio, N., Takamura, N., Krishnaswamy, J., Wood, P., 2018. New policy directions for global pond conservation. Conservation Letters, e12447.

Jaeger, J.A.G., 2000. Landscape division, splitting index, and effective mesh size: new measures of landscape fragmentation. Landscape Ecol. 15, 115-130.

Jarzyna, M.A., Jetz, W., 2018. Taxonomic and functional diversity change is scale dependent. Nature Communications 9, 2565.

Laurance, W.F., Lovejoy, T.E., Vasconcelos, H.L., Bruna, E.M., Didham, R.K., Stouffer, P.C., Gascon, C., Bierregaard, R.O., Laurance, S.G., Sampaio, E. 2002. Ecosystem decay of Amazonian forest fragments: a 22-year investigation. Conservation Biology 16, 605-18.

Levine, J.M., D'Antonio, C.M., 1999. A review of evidence linking diversity and invasibility. Oikos 87 , 15-26.

Mac Nally, R., Lake, P.S., 1999. On the generation of diversity in archipelagos: a re-evaluation of the Quinn-Harrison 'saturation index.' Journal of Biogeography 26, 285-295.

Martin, A.E., 2018. The spatial scale of a species' response to the landscape context depends on which biological response you measure. Current Landscape Ecology Reports 3, 23-33.

Miguet, P., Jackson, H.B., Jackson, N.D., Martin, A.E., Fahrig, L., 2016. What determines the spatial extent of landscape effects on species? Landscape Ecology 31, 1177-1194.

Oficina Nacional Forestal., 2018. Protección de bosque. https://www.onfcr.org/article/proteccion-debosque/

OMNR, 2002. Forest management guide for natural disturbance pattern emulation, Version 3.1. Toronto, Canada, Ontario Ministry of Natural Resources, Queen's Printers for Ontario.

Pautasso, M., 2007. Scale dependence of the correlation between human population presence and vertebrate and plant species richness. Ecology Letters 10, 16-24.

Pfeifer, M., Lefebvre, V., Peres, C.A., Wearn, O., Marsh, C., Banks-Leite, C., Butchart, S., ArroyoRodríguez, V., Barlow, J., Cerezo, A., Cisneros, L., D'Cruze, N., Faria, D:, Hadley, A., Klingbeil, B., Kormann, U., Lens, L., Rangel, G.M., Morante-Filho, J.C., Olivier, P., Peters, S., Pidgeon, A., Ribeiro, D., Scherber, C., Schneider-Maunoury, L., Struebig, M., Urbina-Cardona, N., Watling, J.I., Willig, M., Wood, E., Ewers, R., 2017. Creation of forest edges has a global impact on forest vertebrates. Nature 551, 187-191.

Prevedello, J.A., Gotelli, N.J., Metzger, J.P., 2016. A stochastic model for landscape patterns of biodiversity. Ecological Monographs, 86, 462-479.

Quinn, J.F., Harrison, S.P., 1988. Effects of habitat fragmentation and isolation on species richness: evidence from biogeographic patterns. Oecologia 75, 132-140.

Ramsey, F.L., 1989. Comments on a 'saturation index.' Oecologia 81, 569-570.

Simberloff, D.S., Abele, L.G., 1982. Island biogeography theory and conservation practice. Science 191, 285-286. 
Smith, A.C., Koper, N., Francis, C.M., Fahrig, L., 2009. Confronting collinearity: Comparing methods for disentangling the effects of habitat loss and fragmentation. Landscape Ecology 24, 1271-1285.

Soranno, P.A., Cheruvelil, K.S., Bissell, E.G., Bremigan, M.T., Downing, J.A., Fergus, C.E., Filstrup, C.T., Henry, E.N., Lottig, N.R., Stanley, E.H., Stow, C.A., Tan, P.-N., Wagner, T., Webster, K.E., 2014. Cross-scale interactions: quantifying multi-scaled cause-effect relationships in macrosystems. Frontiers in Ecology and the Environment 12, 65-73.

Taubert, F., Fischer, R., Groeneveld, J., Lehmann, S., Müller, M.S., Rödig, E., Wiegand, T., Huth, A., 2018. Global patterns of tropical forest fragmentation. Nature 554, 519-522.

Taylor, P.D., Fahrig, L., Henein, K., Merriam, G., 1993. Connectivity is a vital element of landscape structure. Oikos 68, 571-573.

Tscharntke, T., Tylianakis, J., Rand, T., Didham, R., Fahrig, L., Batary, P., Bengtsson, J., Clough, Y., Crist, T., Dormann, C., Ewers, R., Holt, R., Holzschuh, A., Klein, A., Kremen, C., Landis, D., Laurance, W., Lindenmayer, D., Scherber, C., Sodhi, N., Steffan-Dewenter, I., Thies, C., vander Putten, W., Westphal, C., 2012. Landscape moderation of biodiversity patterns and processes eight hypotheses. Biological Reviews 87, 661-685.

Tischendorf, L., Fahrig, L., 2000. How should we measure landscape connectivity? Landscape Ecology 15, 633-641.

Trzcinski, M.K., Fahrig, L., Merriam, G., 1999. Independent effects of forest cover and fragmentation on the distribution of forest breeding birds. Ecological Applications 9, 586-593.

Tulloch, A.I.T., Barnes, M.D., Ringma, J., Fuller, R.A., Watson, J.E.M., 2016. Understanding the importance of small patches of habitat for conservation. Journal of Applied Ecology 53, 418-429. 


\section{Figure Captions}

Figure 1. For a given habitat amount (here, 30\% in landscapes A and B, and 15\% in landscapes C and D), habitat fragmentation per se increases with an increase in the number of patches in a landscape of a given size (here, from 3 to 12 patches: A vs. B, and C vs. D). Note that the size of a landscape depends on the particular question and response variable (Miguet et al. 2015, Martin 2018), and the land cover type designated as "habitat" depends on the particular species or species group. To estimate ecological responses to fragmentation independent of habitat amount, landscapes are selected to avoid or minimize the correlation between the amount of habitat and the number of patches across landscapes (e.g., Ethier and Fahrig, 2011; Trzcinski et al., 1999).

Figure 2. Patch-scale studies (A, B, C) document the effects of patch size, patch isolation, or distance to edge on ecological responses, by comparing these responses over multiple patches. Fletcher et al. (2018) extrapolate results of these studies to make inferences about landscape-scale habitat fragmentation effects. These extrapolations are not evidence of landscape-scale fragmentation effects; rather they are predictions that need to be tested using landscape-scale studies (D). Such studies are the subject of the review by Fahrig (2017a).

Figure 3. A wide variety of mechanisms, operating at both patch (P) and landscape (L) scales, may underlie the landscape-scale effects of habitat fragmentation per se on species occurrence, abundance or richness. Some of these mechanisms, emphasized by Fletcher et al. (2018), would predict negative fragmentation effects (red), while others would predict positive fragmentation effects (blue). Fahrig (2003, 2017a) found that effects of fragmentation per se are usually not statistically significant (> 70\% of effects). This implies either that effect sizes are generally small or that both types of mechanisms are often in play, counteracting each other. Fahrig (2017a) also found that $76 \%$ of statistically significant effects of fragmentation per se were positive. This implies that mechanisms leading to positive effects often outweigh mechanisms leading to negative effects of fragmentation. Therefore the observed overall pattern is one of weak effects that are more often positive than negative when significant (thick purple line). 
Figure 1

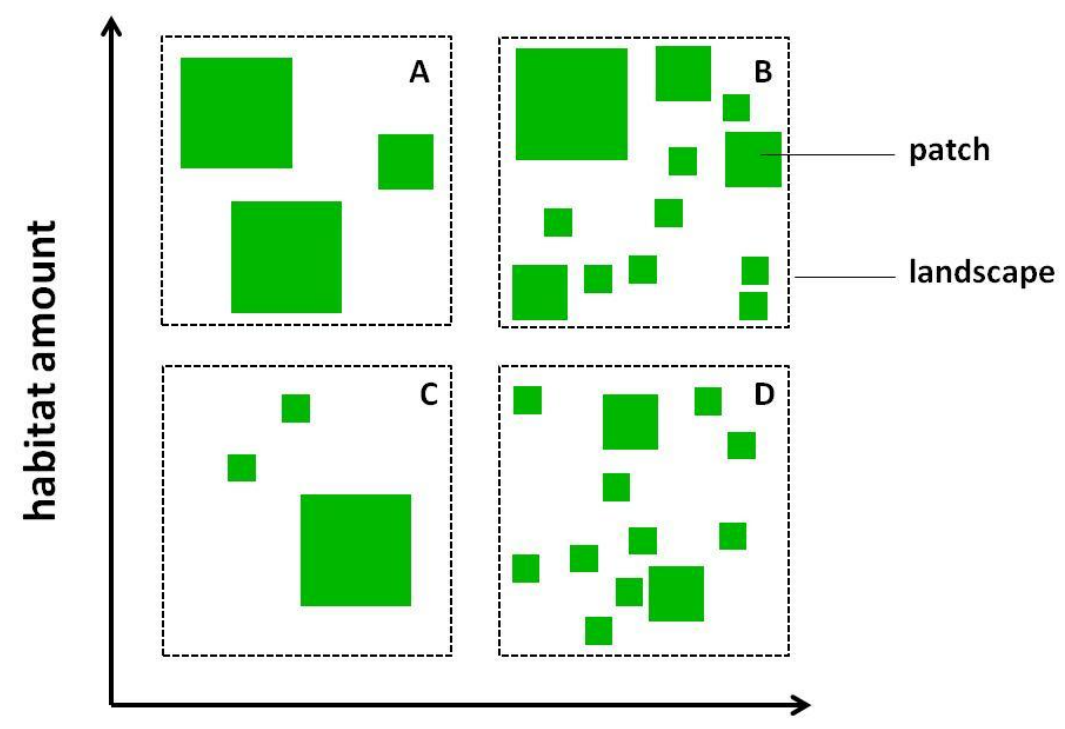

habitat fragmentation per se 
Figure 2
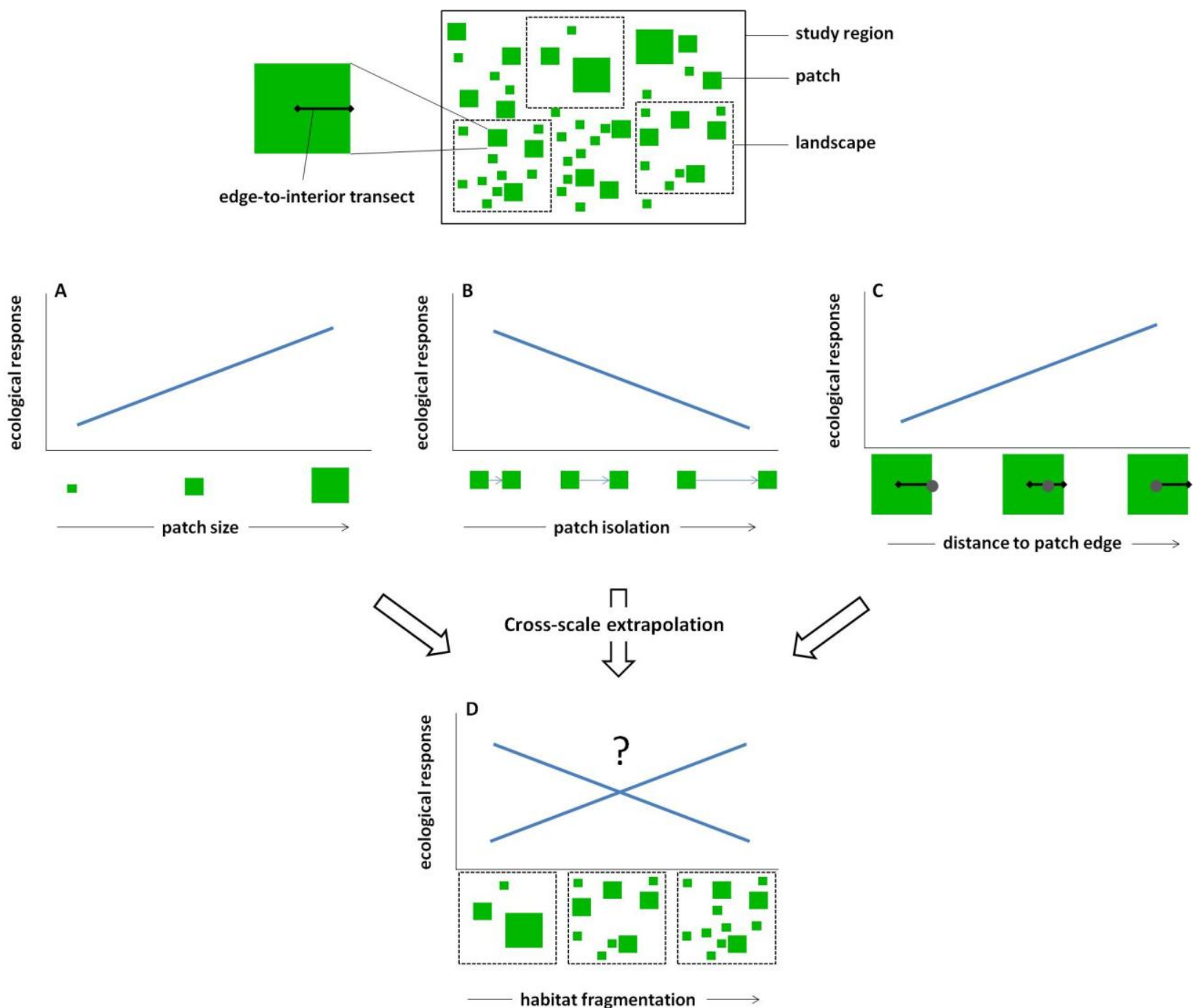
Figure 3

Mechanisms leading to negative effects

- negative edge effects (P)

- increased per-patch extinction rate (P)

- reduced movement success (L)

Mechanisms leading to positive effects

- positive edge effects (P)

- reduced inter- and intra-specific competition (P)

- increased movement success (L)

- spreading of risk (L)

- stabilization of predator-prey or hostparasite interactions (L)

- increased landscape complementation (L)

- higher habitat diversity (L)

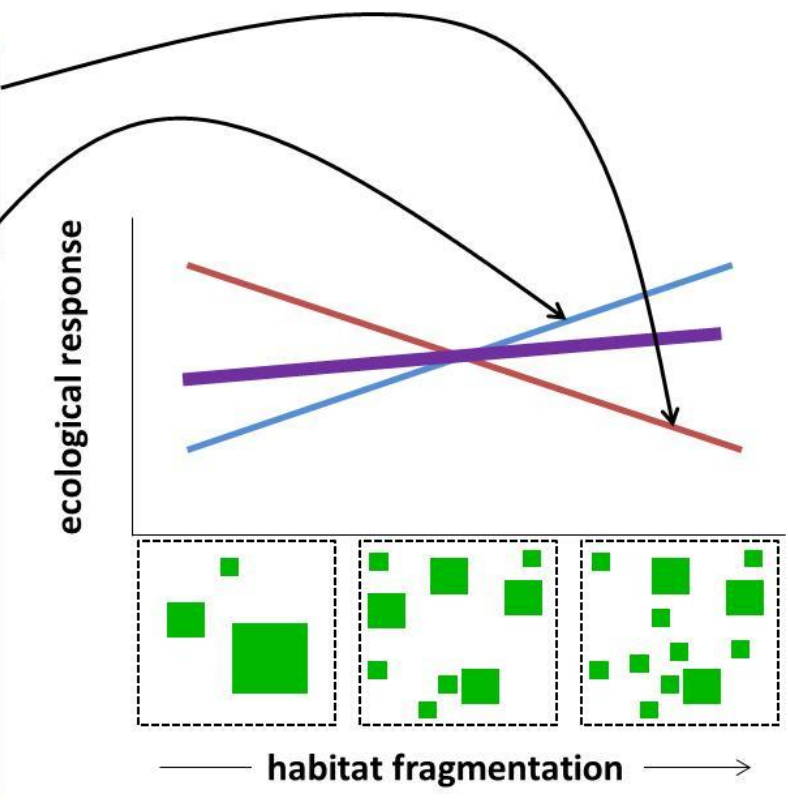

